N. Murakami

\begin{abstract}
Parkinsonism-dementia complex (PDC) is the second most common neurodegenerative disorder in Guam, after amyotrophic lateral sclerosis (ALS). PDC was first described by Hirano 1961. A familial appearance is
\end{abstract}

Nobuyuki Murakami (两) Department of Neurology Kariya General Hospital Toyota Medical Corporation 5-15, Sumiyoshi-cho, Kariya City Aichi 448-8505, Japan

\title{
Parkinsonism-dementia complex on Guam - Overview of clinical aspects
}

seen among some PDC cases, which may also include ALS, and vice versa, but subsequent research including pedigree analysis, prospective case control registries, and the search for specific gene markers has failed to yield a satisfactory genetic explanation. Important diagnostic indicators of the illness include rigido-akinetic type Parkinsonism and severe dementia.

In PDC, rigidity is so marked that postural deformities such as a generally flexed posture become rather prominent. Gait disturbances are a common initial symptom. Hyperreflexia and spinal muscular atrophy, developing mainly in the distal extremities, are frequently observed. These mixedsyndrome patients can be seen as clear support for the view that Guam ALS and PDC constitute a single mixed disease entity with a spectrum of clinical expression.

The present paper offers an overview and description of the clinical features of PDC.

Key words Parkinsonism-dementia complex (PDC) · Amyotrophic lateral sclerosis (ALS) · Lytico-Bodig

\section{Introduction}

Parkinsonism-dementia complex (PDC) is a unique, fatal disease of the CNS which was not clearly recognized as a disease entity before the description by Hirano in $1961[4,5]$. PDC is now known as Hirano's disease.

During an island-wide survey of amyotrophic lateral sclerosis (ALS) in 1953, Mulder and Kurland came across 22 patients with masked faces, bradykinesia, cogwheel rigidity, dysarthria, and severe cognitive dysfunction. They believed this curious syndrome to be post-encephalitic in origin, perhaps similar to the sequela of Western equine encephalitis [6, 7].

Over the past 50 years, several advances have been made in the understanding of PDC. These findings have revealed that prior conceptions of the neurological site, epidemiology, neuropathological development, and approach to the treatment of the Parkinsonism of PDC were based on dubious assumptions.

\section{History}

The Chamorro people may have in earlier times regarded PDC as a part of the aging process, since early symptoms often mimic the changes associated with advancing years. Several terms have been relatively recently applied to PDC, including "rayput", meaning loafer or lazy person. At present, the name used most commonly is "bodig". This term arose from a Chamorro family who owned a store or "bodega" on Saipan prior to 1914. A Chamorro employee at the bodega developed PDC and was sent home to Guam where he was nicknamed "bodig sihig", or sick fellow from the bodega [2].

The first clinical description of Guamanian PDC was made in 1936 by Okaya, a Japanese physician. He reported a Chamorro patient with symptoms thought to be paralysis agitans. In retrospect, this appears to be the first description of PDC. Yase et al. translated Okaya's Japanese paper into English in 1978 [9]. 
Since the above-mentioned island-wide survey of ALS in 1953, many neurologists, neuropathologists, neuroepidemiologists, and others have visited Guam and conducted extensive studies on ALS and PDC, which are called Lytico-Bodig among the Chamorro population of Guam.

Clinical and epidemiologic studies from 1949 to 1954 demonstrated a phenomenal frequency of ALS (Lytico) on Guam; its incidence, prevalence, and mortality rates were estimated to be about 50-100 times as high as those reported elsewhere. It became evident that the Chamorro people on Guam also had a high incidence of one form of PDC (Bodig). Both ALS and PDC (Lytico-Bodig) occurred among the same geographically isolated population and involved similar age groups with similar prognoses and considerable overlap with regard to symptomatology and familial aggregation. This led to the suspicion that the two diseases were related $[2,4,5]$.

\section{Clinical findings}

In 1980, Murakami reported the average age at onset of PDC among 70 cases to be $58.9 \pm 8.3$ years, whereas the average age in Hirano's report of 1961 was 53 years (Table 1). The average age at onset of PDC has thus increased since Hirano's report in 1961 and Elizam's in 1966 [3].

The sex ratio for PDC in Murakami's report in 1980 was 1.8:1, against 3:1 in Hirano's report. Sixty percent of patients with PDC are males, and this male dominance is a characteristic of the disease. However, the sex ratio has been found to be closer now than in the past [8].

The durations of the disease among 70 cases ranged from 1 to 15 years, with an average duration of $5.0 \pm 2.6$ years (Tables 2,3). This suggests that the progression of PDC is relatively rapid compared to Parkinson's or Alzheimer's disease.

Table 1 Age of onset

\begin{tabular}{|c|c|c|c|c|c|c|}
\hline & \multicolumn{3}{|c|}{ Guam ALS } & \multicolumn{3}{|c|}{ Guam PD } \\
\hline & Male & Female & Total & Male & Female & Total \\
\hline No. & 43 & 27 & 70 & 45 & 25 & 70 \\
\hline Mean (yr.) & 51.2 & 47.8 & 49.9 & 56.9 & 62.4 & 58.9 \\
\hline S.D. (yr.) & 8.6 & 10.7 & 9.6 & 7.4 & 8.9 & 8.3 \\
\hline
\end{tabular}

Table 2 Duration

\begin{tabular}{lcccccc}
\hline & \multicolumn{3}{c}{ Guam ALS } & \multicolumn{3}{c}{ Guam PD } \\
& Male & Female & Total & Male & Female & Total \\
\hline No. & 43 & 27 & 70 & 45 & 25 & 70 \\
Mean (yr.) & 4.7 & 5.9 & 5.1 & 5.2 & 4.6 & 5.0 \\
S.D. (yr.) & 3.5 & 5.1 & 4.2 & 2.8 & 2.2 & 2.6 \\
\hline
\end{tabular}

Table 3 Distribution of duration

\begin{tabular}{lrlrl}
\hline Duration & Guam ALS (Elizan) & \multicolumn{3}{c}{ Guam ALS (Murakami) } \\
\hline$\leq 2$ & 28 & $(41.8 \%)$ & 24 & $(34.3 \%)$ \\
$3-4$ & 17 & $(25.4 \%)$ & 25 & $(35.7 \%)$ \\
$5-9$ & 16 & $(23.9 \%)$ & 12 & $(17.1 \%)$ \\
$10 \geq$ & 6 & $(9.0 \%)$ & 9 & $(12.9 \%)$ \\
Total & 67 & $(100.0 \%)$ & 70 & $(100.0 \%)$ \\
\hline Duration & Guam PD (Elizan) & \multicolumn{2}{c}{ Guam PD (Murakami) } \\
\hline$\leq 2$ & 15 & $(25.9 \%)$ & 13 & $(18.6 \%)$ \\
$3-6$ & 35 & $(60.3 \%)$ & 44 & $(62.9 \%)$ \\
$7 \geq$ & 8 & $(13.8 \%)$ & 13 & $(18.6 \%)$ \\
Total & 58 & $(100.0 \%)$ & 70 & $(100.0 \%)$ \\
\hline
\end{tabular}

From Elizan, 1966; Murakami, 1980

\section{Clinical features}

Initial symptoms may be those of Parkinsonism, dementia, or both. Some patients manifest progressive dementia without extrapyramidal dysfunction until late in the course, although most PDC patients show a gait disturbance as an initial symptom, in addition to various other extrapyramidal dysfunctions. This gait disturbance is due to bradykinesia, rigidity and impaired postural reflexes.

Dementia in PDC is characterized by a progressive dulling of all intellectual faculties, recent memory deficits, disorientation in time, place and then person, as well as personality and behavioral changes. Finally, PDC patients reach a bedridden state with pelvicrural flexion posture and severe contractures in various joints.

Most PDC patients show bradykinesia and rigidity similar to what is seen with Parkinson's disease, but more marked. Generalized motor slowness and profound difficulty initiating volitional movements and in associated movements develop in all patients. Plastic "lead-pipe" rigidity is the most prominent sign of extrapyramidal dysfunction. Both axial and distal rigidities appear, but few cases exhibit pill-rolling type tremors.

Dysarthria and dysphagia occur in all PDC patients, usually as a result of both extrapyramidal and cortico-bulbar dysfunction, compounded by advancing dementia [2].

Masked, oily faces and reduced blinking develop in all PDC patients. Ocular motility usually remains intact, although in rare cases vertical gaze, especially upward, ultimately becomes impaired.

As the disease progresses, ambulation deteriorates due to the combined influences of rigidity, akinesia, and the loss of postural reflexes. Festination and propulsion are not common features of PDC, possibly because they are masked by the limb deformities and gait apraxias characteristic of this disease [2]. 
Hirano classified PDC into 3 groups: 1) Parkinsonismdementia complex, 2) Parkinsonism dementia with clinical evidence of upper motor neuron disease, and 3) Parkinsonismdementia with clinical evidence of upper and lower motor neuron disease (ALS). PDC may evidence such signs of motor neuron involvement as multisegmental muscle atrophy, spasticity, hyperactive deep tendon reflexes, and, occasionally, fasciculations. PDC showing upper and lower motor neuron involvements is clinically distinct from ALS.

\section{Differential diagnosis}

PDC must be diagnosed so as to differentiate it from other disorders such as Parkinson's disease, Alzheimer's disease, progressive supranuclear palsy (PSP), corticobasal degeneration (CBD), presenile dementia with motor neuron disease, diffuse neurofibrillary tangles with calcification (PNTC), and dementia with Lewy bodies (DLB). The progression of PDC is relatively rapid compared to Parkinson's or Alzheimer's diseases. Some cases of both ALS and PDC show familial clustering. These cases occasionally present with the same clinical picture and response to levodopa in the early stage.

\section{Concluding remarks}

According to Chen in 1998 [1], the questions yet to be answered are 1) Is ALS/PDC (Lytico-Bodig) a single disease entity with a spectrum of expression? 2) Is there a genetic predisposition at the molecular level? Is a mitDNA point mutation operative in ALS/PDC? 3) What is the mode of entry of the relevant etiological agent into the CNS? Is it gastroenteric mucosa or respiratory epithelium? 4) What triggers free radicals and oxidative injury to the neurons?

While there are many hypotheses and proposed future strategies with regard to Guam ALS/PDC, it is clear that we are far from understanding the underlying cause. The answer to this mystery remains a riddle.

\section{References}

1. Chen KM: The history of Guam ALS/PDC, 1900-1998 (1998) Annotated chronological bibliography

2. Chen KM, Chase TN (1985) Parkinsonismdementia. In: Vinken PJ, Bruyn GW (eds) Handbook of Clinical Neurology. Extrapyramidal Disorders, Elsevier Science, Amsterdam 49: 167-183

3. Elizan TS, Hirano A, Abrams BM et al. (1966) Amyotrophic lateral sclerosis and Parkinsonism-dementia complex of Guam: Neurologic reevaluation. Arch Neurol 14: 356-368
4. Hirano A, Kurland LT, Krooth RS, Lessel S (1961) Parkinsonism-dementia complex: an endemic disease on the island of Guam. I. Clinical Features. Brain 84: 642-661

5. Hirano A, Malamud N, Kurland LT (1961) Parkinsonism-dementia complex: an endemic disease on the island of Guam. II. Pathological Features. Brain 84: 662-679

6. Kurland LT, Mulder DW (1954) Epidemiologic investigations of amyotrophic lateral sclerosis. I. Preliminary report on geographic distribution, with special reference to Mariana Islands, including clinical and pathologic observation. Neurology 4: 355-378
7. Mulder DW, Kurland LT, Irriarte LLG (1954) Neurologic disease on the island of Guam. U.S. Armed Forces Med J 5: 1724-1739

8. Murakami N (1980) A study on the natural history of amyotrophic lateral sclerosis in Japan and Guam. Nagoya-Igaku 103: 154-163

9. Yase Y, Chen KM, Brody JA, Toyokura Y (1978) An historical note on the Parkinsonism-dementia complex of Guam. Neuro Med (Tokyo) 8: 583-589 